\title{
Sintomas depressivos em idosos: estudo transversal de base populacional
}

\author{
Depressive symptoms among the elderly: \\ a cross-sectional population-based study
}

Natália Hellwig ${ }^{1}$

Tiago Neuenfeld Munhoz ${ }^{1}$

Elaine Tomasi ${ }^{1}$
${ }^{1}$ Programa de Pós-

Graduação em

Epidemiologia, Faculdade

de Medicina,

Universidade Federal

de Pelotas. R. Marechal

Deodoro 1160, Centro.

96020-220 Pelotas RS

Brasil.natalia.hellwig@ gmail.com
Abstract A cross-sectional population-based study was conducted in Pelotas, Rio Grande do Sul, in 2014 with the aim of measuring the prevalence and factors associated with depressive symptoms among the elderly. Sampling was carried out by clusters in two stages. All the elderly people ( $\geq$ 60 years) living in the selected households were invited to participate. The occurrence of depressive symptoms was measured using GDS-10 (Geriatric Depression Scale) with $\geq 5$ cut-off. Information for 1,451 elderly people was obtained. The prevalence of depressive symptoms was $15.2 \%$ (95\% CI 13.2 to 17.2). After multivariate analysis, the occurrence of depressive symptoms was higher among women, the elderly in economically straitened circumstances, those who were out of work, the physically inactive, those with worse self-reported health and those with functional disability. Greater attention should be given to the identification of depressive symptoms among the elderly and associated factors to inform policy and planning interventions for treatment and management of this ailment at the collective level.

Key words Depression, Elderly, Cross-sectional studies, Mental health, Health of the elderly
Resumo Foi realizado um estudo transversal de base populacional na cidade de Pelotas, Rio Grande do Sul, em 2014, com o objetivo de medir a prevalência e identificar os fatores associados aos sintomas depressivos em idosos. A amostragem foi realizada por conglomerados em dois estágios. Todos os idosos ( $\geq 60$ anos) residentes nos domicílios selecionados foram convidados a participar. A ocorrência dos sintomas depressivos foi medida utilizando-se a GDS-10 (Geriatric Depression Scale) com o ponto de corte $\geq 5$. Foram obtidas informações de 1.451 idosos. A prevalência dos sintomas depressivos foi de 15,2\% (IC95\% 13,2-17,2). Após análise multivariável, a ocorrência de sintomas depressivos foi maior entre as mulheres, os idosos de pior situação econômica, aqueles que não trabalhavam, os fisicamente inativos, aqueles com pior autoavaliação de saúde e naqueles com incapacidade funcional. Maior atenção deve ser dada à identificação de sintomas depressivos em idosose seus fatores associados para fundamentar politicas e planejamentos de intervenções para tratamento e manejo desta doença em nível coletivo.

Palavras-chave Depressão, Idoso, Estudos transversais, Saúde mental, Saúde do idoso 


\section{Introdução}

O aumento da expectativa de vida da população, somado à queda nas taxas de fecundidade e mortalidade, é responsável pelo crescimento da proporção de idosos no Brasil ${ }^{1}$. Segundo estimativa do Instituto Brasileiro de Geografia e Estatística (IBGE), o número de idosos deve passar de 14,9 milhões (7,4\% da população) em 2013, para 58,4 milhões, correspondendo a $26,7 \%$ da população, em $2060^{2}$.

Esse envelhecimento populacional é acompanhado pelo aumento da incidência de doenças crônicas não transmissíveis na população de modo geral, incluindo alguns transtornos mentais, principalmente os demenciais e os depressivos $^{3}$. A depressão é uma das doenças de maior prevalência entre os idosos, com grande impacto na vida do sujeito acometido, possivelmente sendo a causa mais frequente de diminuição na qualidade de vida nesta faixa etária ${ }^{4,5}$. Ela pode ter consequências graves, além do sofrimento psíquico dos próprios idosos e da família, como prejuízos nos relacionamentos interpessoais e no desempenho de atividades cotidianas, incapacidade relacionada à doença e aumento dos custos em saúde ${ }^{6}$.

A prevalência de depressão nos idosos pode diferir substancialmente dependendo da definição utilizada para sua operacionalização, do instrumento usado para sua detecção e, principalmente, da população estudada. Estudos de base populacional, conduzidos em diferentes países, observaram prevalências de sintomas depressivos que variaram entre $6,1 \%^{7}$ até $38,5 \%{ }^{8}$. Entre os indivíduos mais suscetíveis à ocorrência de depressão estão as mulheres, os idosos com situação econômica desfavorável, aqueles com história de eventos estressores na vida, idosos com percepção de baixa qualidade de vida e condições de saúde, aqueles com alguma limitação funcional, e os acometidos por doenças crônicas, dor e comorbidades $^{9-11}$.

O entendimento do estado de saúde mental dos idosos e seus fatores associados podem contribuir com subsídios para o planejamento e para as políticas de atenção que visem o envelhecimento saudável. Estudos epidemiológicos que apresentem estimativas populacionais consistentes a respeito da depressão na população idosa e seus possíveis determinantes, apesar de sua importância, são relativamente escassos na literatura mundial e, principalmente, no Brasil. Este estudo teve como objetivo estimar a ocorrência de sintomas depressivos em indivíduos com 60 anos ou mais e identificar os fatores associados em uma amostra representativa dos idosos residentes na cidade de Pelotas, Rio Grande do Sul, Brasil.

\section{Métodos}

Este estudo fez parte do Consórcio de Mestrado Orientado para a Valorização da Atenção ao Idoso - COMO VAI?, um estudo transversal, de base populacional, realizado entre janeiro e agosto de 2014 na zona urbana de Pelotas, Rio Grande do Sul, município com 328.275 habitantes (Censo Demográfico de 2010) ${ }^{2}$. O estudo incluiu projetos de 18 mestrandos do Programa de PósGraduação em Epidemiologia da Universidade Federal de Pelotas - UFPEL, e avaliou diferentes desfechos relacionados à saúde dos idosos.

O cálculo do tamanho amostral para o estudo de prevalência considerou uma população de 46.000 idosos, prevalência de 20\% do desfecho, nível de confiança de 95\%, margem de erro de três pontos percentuais e efeito de delineamento de 1,5 . Com acréscimo de $10 \%$ para perdas, o tamanho amostral necessário foi de 1.112 idosos. Para o estudo de associação, considerou-se um poder de $80 \%$, nível de significância de $95 \%$, razão de 40/60 entre não expostos e expostos, prevalência de $18 \%$ nos não expostos (sexo masculino), razão de prevalências de 1,5 e efeito de delineamento de 1,5. Adicionou-se, ainda, 10\% para perdas e $15 \%$ para controle de possíveis fatores de confusão, sendo de 1.450 idosos a amostra necessária.

O processo de amostragem foi realizado em dois estágios, tendo como unidades amostrais primárias os setores censitários ${ }^{2}$. Foram ordenados os 488 setores da zona urbana de acordo com a renda média familiar e, posteriormente, a partir de um primeiro setor definido aleatoriamente, foram selecionados sistematicamente 133. Dentre os domicílios habitados, foram selecionados de forma sistemática aqueles que iriam compor a amostra, obtendo-se 31 domicílios por setor, resultando em 4.123 domicílios. Todos os indivíduos com 60 anos ou mais residentes nestes domicílios foram convidados a participar, excluindo-se aqueles com incapacidade cognitiva ou mental, os hospitalizados e os institucionalizados.

O instrumento utilizado para este estudo foi um questionário padronizado com informações sobre características demográficas, socioeconômicas, comportamentais e de saúde. A coleta de dados foi realizada nos domicílios por entrevistadoras treinadas e padronizadas. Para medir a 
prevalência do desfecho, foi utilizada a Escala de Depressão Geriátrica (Geriatric Depression Scale - GDS $)^{12}$, versão brasileira abreviada ${ }^{13}$. Este instrumento de rastreamento contém 10 itens com respostas do tipo "sim ou não", e se refere a um período recordatório de sete dias anteriores à entrevista. Para cada resposta se atribuiu um ponto, cuja soma resultou em um escore variando entre 0 e 10. Foram considerados com sintomas depressivos os idosos com escore $\geq 5$. Este instrumento com este ponto de corte foi escolhido por apresentar bons indicadores de sensibilidade e especificidade: $80,5 \%$ e $78,3 \%$, respectivamente, de acordo com os critérios da CID-10 para Episódio Depressivo Maior; e 84,8\% e 67,7\%, respectivamente, de acordo com os critérios do DSM-IV para Transtorno Depressivo Maior ${ }^{13}$.

As variáveis demográficas e socioeconômicas avaliadas foram sexo (masculino, feminino), idade (60-64, 65-69, 70-74, 75-79, 80 ou mais), situação conjugal (com companheiro/a, sem companheiro/a), escolaridade em anos completos de estudo (nenhum, 1 a 3, 4 a 7, 8 a 10, 11 ou mais), classe econômica, de acordo com a Classificação Econômica da Associação Brasileira de Empresas de Pesquisa (ABEP), categorizada em classes $\mathrm{A} / \mathrm{B}, \mathrm{C}, \mathrm{D} / \mathrm{E}$, e trabalho atual (sim, não), sendo considerado como trabalho qualquer emprego remunerado (profissional liberal, salário fixo ou trabalho informal) exercido pelo idoso. As variáveis comportamentais foram tabagismo atual ( sim, não), sendo classificados como tabagistas os que relataram ter utilizado, pelo menos, um cigarro por dia há mais de um mês; uso de bebida alcoólica (sim, não), sendo classificado como uso de álcool a ingestão de qualquer dose nos trinta dias anteriores à entrevista. $\mathrm{O}$ nível de atividade física (ativo ou insuficientemente ativo) foi mensurado pelo International Physical Activity Questionnaire (IPAQ) ${ }^{14}$ (foram classificados como ativos aqueles que relataram $\geq 150$ minutos semanais de atividades físicas no lazer). As variáveis de saúde foram autopercepção de saúde (muito boa/boa, regular, ruim/muito ruim); morbidade autorreferida (nenhuma, uma, duas, três), a partir do relato de diagnóstico médico de hipertensão arterial, diabetes mellitus e doença cardíaca. A capacidade funcional foi avaliada pela Escala de Independência em Atividades da Vida Diária $(\text { Escala de Katz })^{15}$, e os idosos foram classificados em independentes (não necessitavam de ajuda para realizar nenhuma atividade) ou dependentes (necessitavam de ajuda para realizar, pelo menos, uma atividade).
O instrumento de pesquisa foi programado eletronicamente no software Pendragon 6.1. Utilizaram-se netbooks para a coleta dos dados, possibilitando a entrada das informações diretamente no servidor de dados, evitando erros de digitação e de codificação dos questionários, além de possibilitar a realização de análises de inconsistências. Para identificar possíveis inconsistências, foi realizada semanalmente a checagem do banco de dados. Para controle de qualidade dos dados, uma versão reduzida do questionário foi reaplicada pelos supervisores de campo em 10\% da amostra, selecionada aleatoriamente. A variável escolaridade foi utilizada para o cálculo de concordância, e obtevese um coeficiente Kappa ponderado de 0,83.

Para o tratamento e a análise dos dados foi utilizado o programa Stata 12.0. O efeito de delineamento decorrente do processo de amostragem complexa foi levado em consideração em todas as análises, utilizando-se o prefixo svy. Foi utilizada regressão de Poisson para estimar as razões de prevalência (RP) e obter testes de heterogeneidade e de tendência linear nas análises brutas e ajustadas, com nível de significância de 5\%. Em epidemiologia, a RP é a medida de associação indicada para estudos transversais que avaliam desfechos binários, apesar de os pacotes estatísticos não disporem de um método de regressão no qual se possa estimar a RP. Portanto, tradicionalmente se utiliza a regressão logística para estudo de desfechos binários, estimando-se a razão de odds (OR), uma vez que esta abordagem está disponível em diferentes pacotes estatísticos. No entanto, Barros e Hirakata ${ }^{16}$ desenvolveram uma abordagem estatística que permite a estimação da RP em estudos transversais, sendo esta abordagem utilizada no atual estudo. A análise ajustada utilizou um modelo hierárquico de determinação ${ }^{17}$. Este tipo de análise considera o efeito de cada variável em relação ao desfecho, controlando para possível confusão entre as variáveis do mesmo nível e as de níveis superiores. Foi utilizado como critério de inclusão no modelo um valor $\mathrm{p}<0,20$ na análise bruta, e utilizado o mesmo valor p como critério de manutenção das variáveis em cada nível na análise ajustada.

O estudo foi aprovado pelo Comitê de Ética em Pesquisa da Faculdade de Medicina da UFPEL. Todos os participantes assinaram o Termo de Consentimento Livre e Esclarecido antes da coleta das informações. Os idosos identificados com risco grave de depressão foram atendidos no domicílio por uma psicóloga e encaminhados para os serviços de saúde do município. 


\section{Resultados}

Foram visitados 3.799 domicílios, representando $92,1 \%$ do total de domicílios elegíveis e, dentre estes, 1.379 eram habitados por pelo menos um idoso. Foram localizados 1.844 idosos, e entrevistados 1.451 , sendo contabilizadas $9,8 \%$ de perdas e 11,6\% de recusas. Há informações de 1.394 idosos para o desfecho. $\mathrm{O}$ efeito de delineamento foi de 1,1 .

A Tabela 1 apresenta as características da população estudada. A maior parte da amostra foi composta por mulheres $(63,2 \%)$ e pouco mais da metade dos idosos tinha entre 60 e 69 anos (53\%). Aproximadamente um terço $(36,7 \%)$ tinha até três anos completos de estudo, 57,3\% pertenciam à Classe $\mathrm{C}, 53,4 \%$ viviam com companheiro, e $80,1 \%$ referiram não estar trabalhando na época da entrevista. Em relação às variáveis comportamentais, $12,8 \%$ eram tabagistas, $21,9 \%$ referiram ter consumido alguma bebida alcoólica nos 30 dias anteriores à entrevista e 18,7\% relataram praticar 150 minutos ou mais de atividades físicas de lazer por semana. Mais da metade dos idosos considerou sua saúde boa ou muito boa $(53,8 \%)$, cerca de um terço tinha ao menos uma doença crônica $(37,3 \%)$ e foram considerados dependentes para a realização de ao menos uma atividade da vida diária (34,7\%).

A prevalência de sintomas depressivos foi de 15,2\% (IC95\% 13,2-17,2). A Tabela 2 apresenta as análises bruta e ajustada para a associação entre as variáveis independentes e o desfecho. $\mathrm{Na}$ análise bruta, as mulheres, os idosos de menor escolaridade, os de menor classe econômica, aqueles que viviam sem companheiro, os que não trabalhavam, aqueles que referiram não ter ingerido bebida alcoólica nos 30 dias anteriores à entrevista, os insuficientemente ativos, aqueles com pior autopercepção de saúde, os que referiram ter alguma doença crônica e aqueles considerados dependentes para a realização de alguma atividade da vida diária apresentaram maiores prevalências de sintomas depressivos. Somente a idade e o tabagismo não apresentaram associação com o desfecho.

A análise ajustada mostrou que as mulheres apresentaram quase duas vezes mais sintomas depressivos do que os homens $(\mathrm{RP}=1,90$; IC95\% 1,42-2,55). Os sintomas depressivos foram $80 \%$ maios prevalentes nos idosos de pior condição socioeconômica (classes D e E) quando comparados àqueles de melhor condição socioeconômica ( $\mathrm{RP}=1,78$; IC95\% 1,16-2,74). Os idosos que não trabalhavam apresentaram maior prevalência de sintomas depressivos se comparados aos que trabalhavam ( $\mathrm{RP}=1,57$; IC95\% 1,022,42 ). Foi observada maior prevalência de sintomas depressivos nos idosos fisicamente inativos quando comparados aos que referiram praticar 150 minutos ou mais de atividades físicas semanais no lazer $(\mathrm{RP}=1,97$; IC95\% 1,12-3,47). Os sintomas depressivos foram maiores entre os idosos que avaliaram sua saúde como regular ( RP = 2,76, IC95\% 1,82-4,19) ou ruim/muito ruim (RP = 5,78; IC95\% 3,69-9,04) em relação aos com melhor autopercepção de saúde. Aqueles idosos classificados como dependentes para a realização de atividades da vida diária tiveram mais sintomas depressivos se comparados aos independentes $(\mathrm{RP}=1,68$; IC95\% 1,30-2,18).

\section{Discussão}

Este estudo identificou que 15,2\% dos idosos da cidade de Pelotas (RS) apresentaram sintomas depressivos. Os grupos populacionais com maior ocorrência de sintomas depressivos foram as mulheres, os idosos de pior situação econômica, os que não trabalhavam, os fisicamente inativos, aqueles que se consideravam com pior saúde, e aqueles com incapacidade funcional. A escolaridade, a situação conjugal, o uso de álcool, o uso de tabaco e a presença de doenças crônicas não se mantiveram associadas à ocorrência de sintomas depressivos na população idosa após ajuste.

Um estudo de revisão sistemática e metanálise apontou que idosos que vivem na comunidade apresentaram prevalências variando desde 13\% até $39 \%{ }^{18}$. Estudos de base populacional conduzidos no Brasil utilizando a GDS-15 encontraram prevalências de 20 a 30\% de depressão nesta faixa etária ${ }^{19-24}$. As diferenças encontradas nas prevalências entre os estudos são plausivelmente explicadas pela diferença entre as metodologias dos estudos e de diferenças nas características da amostra. Soma-se a isto o fato de que a utilização de diferentes instrumentos, pontos de corte utilizados para a definição de depressão e as diferenças nos resultados da validade das versões da escala GDS são fatores que influenciariam a prevalência do desfecho, mesmo se aplicados à mesma amostra. Estudos de validação da GDS10 e GDS-15 utilizaram o mesmo padrão-ouro para a definição do desfecho, de acordo com os critérios da CID-10 e do DSM-IV. No entanto, a GDS-10 apresentou menor sensibilidade e maior especificidade para a detecção de casos. Entende-se que quanto menor a sensibilidade de um 
Tabela 1. Descrição da amostra e prevalência de sintomas depressivos segundo variáveis demográficas, socioeconômicas, comportamentais e de saúde na população idosa de Pelotas, Brasil. 2014. (n = 1.394)

\begin{tabular}{|c|c|c|}
\hline Variáveis & n (\%) & Prevalência de depressão (IC95\%) \\
\hline \multicolumn{3}{|l|}{ Sexo } \\
\hline Masculino & $513(36,8)$ & $9,9(7,3-12,5)$ \\
\hline Feminino & $881(63,2)$ & $18,3(15,7-20,8)$ \\
\hline \multicolumn{3}{|l|}{ Idade } \\
\hline 60 a 64 & $383(27,5)$ & $14,4(10,8-17,9)$ \\
\hline 65 a 69 & $354(25,5)$ & $16,7(12,8-20,6)$ \\
\hline 70 a 74 & $263(18,9)$ & $14,1(9,8-18,3)$ \\
\hline 75 a 79 & $182(13,1)$ & $18,7(13,0-24,4)$ \\
\hline 80 ou mais & $209(15,0)$ & $12,9(8,3-17,5)$ \\
\hline \multicolumn{3}{|l|}{ Escolaridade em anos completos } \\
\hline Nenhum & $184(13,3)$ & $17,4(11,8-22,9)$ \\
\hline 1 a 3 & $323(23,4)$ & $18,9(14,6-23,2)$ \\
\hline 4 a 7 & $430(31,1)$ & $16,7(13,2-20,3)$ \\
\hline 8 a 10 & $140(10,1)$ & $14,3(8,4-20,2)$ \\
\hline 11 ou mais & $305(22,1)$ & $8,9(5,6-12,1)$ \\
\hline \multicolumn{3}{|l|}{ Classe econômica (ABEP) } \\
\hline $\mathrm{A} / \mathrm{B}$ & $362(27,3)$ & $11,1(8,2-14,0)$ \\
\hline $\mathrm{C}$ & $760(57,3)$ & $15,4(12,7-18,1)$ \\
\hline $\mathrm{D} / \mathrm{E}$ & $204(15,4)$ & $25,0(18,3-31,7)$ \\
\hline \multicolumn{3}{|l|}{ Situação conjugal } \\
\hline Com companheiro(a) & $743(53,4)$ & $12,0(9,6-14,3)$ \\
\hline Sem companheiro(a) & $649(46,6)$ & $19,0(15,9-22,0)$ \\
\hline \multicolumn{3}{|l|}{ Trabalho atual ${ }^{*}$} \\
\hline Sim & $259(19,9)$ & $17,1(14,9-19,4)$ \\
\hline Não & $1.044(80,1)$ & $8,1(4,8-11,5)$ \\
\hline \multicolumn{3}{|l|}{ Fumo atual } \\
\hline Não & $1.214(87,2)$ & $14,6(12,6-16,6)$ \\
\hline Sim & $178(12,8)$ & $19,7(13,8-25,6)$ \\
\hline \multicolumn{3}{|l|}{ Uso de álcool nos últimos 30 dias } \\
\hline $\operatorname{Sim}$ & $305(21,9)$ & $9,5(6,2-12,9)$ \\
\hline Não & $1.085(78,1)$ & $16,9(14,6-19,1)$ \\
\hline \multicolumn{3}{|l|}{ Atividade física (IPAQ) } \\
\hline Ativo(a) ( $\geq 150$ min./sem.) & $253(18,7)$ & $5,1(2,4-7,9)$ \\
\hline Insuficientemente ativo(a) & $1102(81,3)$ & $17,2(15,0-19,5)$ \\
\hline \multicolumn{3}{|l|}{ Autopercepção de saúde } \\
\hline Muito boa/boa & $748(53,8)$ & $5,9(4,2-7,6)$ \\
\hline Regular & $524(37,7)$ & $20,6(17,1-24,1)$ \\
\hline Ruim/muito ruim & $119(8,5)$ & $50,4(41,3-59,5)$ \\
\hline \multicolumn{3}{|l|}{ Morbidade autorreferida ${ }^{¥}$} \\
\hline Nenhuma & $325(23,3)$ & $10,2(6,9-13,5)$ \\
\hline Uma & $557(40,1)$ & $14,7(11,8-17,6)$ \\
\hline Duas & $387(27,8)$ & $16,5(12,8-20,3)$ \\
\hline Três & $122(8,8)$ & $27,0(19,1-35,0)$ \\
\hline \multicolumn{3}{|l|}{ Capacidade funcional (Katz) } \\
\hline Independente & $910(65,3)$ & $9,8(7,8-11,7)$ \\
\hline Dependente & $483(34,7)$ & $25,5(21,6-29,4)$ \\
\hline
\end{tabular}

*Variável com maior número de missings (91). ${ }^{叉}$ Hipertensão arterial, diabetes mellitus e doença cardíaca.

teste, menor a probabilidade de que detecte casos positivos para a doença e que, quanto maior especificidade, maior a probabilidade de detectar não casos. Portanto, a menor sensibilidade e a maior especificidade da GDS-10 em relação à versão de 15 itens poderiam explicar parte desta diferença entre as prevalências observadas nos estudos. Além disso, diversos inquéritos popula- 
Tabela 2. Análise bruta e ajustada para associação entre sintomas depressivos (GDS-10 $\geq 5$ ) e variáveis demográficas, socioeconômicas, comportamentais e de saúde na população idosa de Pelotas, Brasil. 2014. ( $\mathrm{n}=$ 1.394)

\begin{tabular}{|c|c|c|c|c|}
\hline \multirow[b]{2}{*}{ Variáveis } & \multicolumn{2}{|c|}{ Análise bruta } & \multicolumn{2}{|c|}{ Análise ajustada } \\
\hline & RP (IC 95\%) & Valor $\mathrm{p}$ & RP (IC 95\%) & Valor $\mathbf{p}$ \\
\hline \multicolumn{5}{|l|}{ Nível 1} \\
\hline Sexo & & $<0,001$ & & $<0,001$ \\
\hline Masculino & 1 & & 1 & \\
\hline Feminino & $1,84(1,40-2,42)$ & & $1,90(1,42-2,55)$ & \\
\hline Idade & & $0,921 \dagger$ & & \\
\hline 60 a 64 & $1,11(0,72-1,72)$ & & & \\
\hline 65 a 69 & $1,29(0,85-1,97)$ & & & \\
\hline 70 a 74 & $1,09(0,70-1,70)$ & & & \\
\hline 75 a 79 & $1,45(0,89-2,36)$ & & & \\
\hline 80 ou mais & 1 & & & \\
\hline Escolaridade em anos completos & & $0,001^{\dagger}$ & & 0,191 \\
\hline Nenhum & $1,96(1,20-3,22)$ & & $1,52(0,87-2,65)$ & \\
\hline 1 a 3 & $2,13(1,36-3,34)$ & & $1,69(1,01-2,83)$ & \\
\hline 4 a 7 & $1,89(1,20-2,97)$ & & $1,67(1,01-2,77)$ & \\
\hline 8 a 10 & $1,61(0,93-2,79)$ & & $1,64(0,94-2,85)$ & \\
\hline 11 ou mais & 1 & & 1 & \\
\hline Classe econômica (ABEP) & & $<\mathbf{0 , 0 0 1} 1^{\dagger}$ & & 0,011 \\
\hline $\mathrm{A} / \mathrm{B}$ & 1 & & 1 & \\
\hline $\mathrm{C}$ & $1,39(0,99-1,96)$ & & $1,11(0,76-1,63)$ & \\
\hline $\mathrm{D} / \mathrm{E}$ & $2,25(1,56-3,32)$ & & $1,78(1,16-2,74)$ & \\
\hline \multicolumn{5}{|l|}{ Nível 2} \\
\hline Situação conjugal & & 0,001 & & 0,156 \\
\hline Com companheiro(a) & 1 & & 1 & \\
\hline Sem companheiro(a) & $1,58(1,21-2,06)$ & & $1,22(0,92-1,62)$ & \\
\hline Trabalho atual & & $<0,001$ & & 0,043 \\
\hline Sim & 1 & & 1 & \\
\hline Não & $2,11(1,41-3,17)$ & & $1,57(1,02-2,42)$ & \\
\hline \multicolumn{5}{|l|}{ Nível 3} \\
\hline Fumo atual & & 0,061 & & 0,076 \\
\hline Não & 1 & & 1 & \\
\hline Sim & $1,35(0,99-1,84)$ & & $1,37(0,99-1,90)$ & \\
\hline Uso de álcool nos últimos 30 dias & & 0,003 & & 0,57 \\
\hline Sim & 1 & & 1 & \\
\hline Não & $1,77(1,23-2,56)$ & & $1,10(0,77-1,57)$ & \\
\hline Atividade física (IPAQ) & & $<0,001$ & & 0,019 \\
\hline Ativo(a) ( $\geq 150$ min./sem.) & 1 & & 1 & \\
\hline Insuficientemente ativo(a) & $3,36(1,99-5,69)$ & & $1,97(1,12-3,47)$ & \\
\hline Autopercepção de saúde & & $<0,001^{\dagger}$ & & $<0,001$ \\
\hline Muito boa/boa & 1 & & 1 & \\
\hline Regular & $3,50(2,51-4,90)$ & & $2,76(1,82-4,19)$ & \\
\hline Ruim/muito ruim & $8,57(6,07-12,11)$ & & $5,78(3,69-9,04)$ & \\
\hline Morbidade autorreferida $a^{¥}$ & & $<0,001^{\dagger}$ & & 0,787 \\
\hline Nenhuma & 1 & & 1 & \\
\hline Uma & $1,45(0,95-2,20)$ & & $1,28(0,86-1,91)$ & \\
\hline Duas & $1,63(1,11-2,40)$ & & $1,05(0,71-1,54)$ & \\
\hline Três & $2.66(1,68-4,22)$ & & $1,04(0,64-1,68)$ & \\
\hline Capacidade funcional (Escala Katz) & & $<0,001$ & & $<0,001$ \\
\hline Independente & 1 & & 1 & \\
\hline Dependente & $2,60(2,07-3,28)$ & & $1,68(1,30-2,18)$ & \\
\hline
\end{tabular}

\footnotetext{
† Teste Wald de tendência linear. ${ }^{¥}$ Hipertensão arterial, diabetes mellitus e doença cardíaca.
} 
cionais realizados em outros países, utilizando as diferentes versões da GDS (5, 10, 15 ou 30 itens), obtiveram prevalências que variaram entre $6,1 \%{ }^{7}$ e $27,8 \%{ }^{25}$.

Os achados deste estudo estão de acordo com as características mais consistentemente associadas à ocorrência de sintomas depressivos encontrada na literatura científica. Dentre os principais fatores associados está o sexo feminino. Estudos realizados em diversos países ${ }^{8,26-30}$ têm observado esta associação em diferentes faixas etárias. Contudo, as explicações causais para esta diferença ainda são inconsistentes. É possível que as mulheres sejam mais suscetíveis a eventos potencialmente estressores, que são determinados por papeis sociais e de gênero, o que pode influenciar na ocorrência de sintomas depressivos ${ }^{31}$. Além disso, entre as mulheres idosas, a privação de estrogênio também é apontada como um fator de risco para a ocorrência de depressão ${ }^{32}$.

O nível socioeconômico se mostra associado à ocorrência de depressão em diferentes países ${ }^{33}$. $\mathrm{O}$ presente estudo observou maior prevalência de sintomas depressivos entre aqueles com pior condição socioeconômica quando os comparamos aos idosos pertencentes às classes economicamente mais favorecidas. No entanto, o nível de escolaridade não se mostrou associado ao desfecho, contrariando o observado em alguns estu$\operatorname{dos}^{25,34}$. Uma meta-análise apontou que o baixo nível socioeconômico está geralmente associado com alta morbidade psiquiátrica, incapacidade e falta de acesso a cuidados de saúde. Dificuldades financeiras podem gerar ansiedade e preocupações que, somadas a dificuldades de acesso a serviços de saúde, podem contribuir tanto para o surgimento quanto para a manutenção de quadros depressivos ${ }^{35}$.

Os idosos que não trabalhavam também apresentaram maior prevalência de sintomas depressivos quando comparados aos que trabalhavam. Embora haja forte evidência de bidirecionalidade nesta associação, uma vez que a depressão é uma das doenças crônicas mais incapacitantes no mundo ${ }^{36}$, cabe destacar os resultados observados em um estudo conduzido por Gazzale et al. ${ }^{37}$, que identificou maior ocorrência média de sintomas depressivos em idosos sem atividade de trabalho remunerada, e atribuiu isto à desvalorização que o idoso sofre na sociedade. Principalmente nos países de média e baixa renda, é possível que aqueles idosos que se mantêm no mercado de trabalho se sintam mais úteis à sociedade.

Ainda, este estudo indicou maior prevalência de sintomas depressivos em idosos insuficiente- mente ativos. A produção científica tem evidenciado uma associação positiva entre a prática regular de atividade física e melhor condição de saúde mental ${ }^{38,39}$. Uma revisão sistemática apontou que a prática de atividade física em idosos favorece a interação social e proporciona uma maior sensação de controle do indivíduo sobre os eventos e as demandas do meio ${ }^{39}$. O estudo conduzido por Mather et $\mathrm{al}^{38}$ apontou que o efeito benéfico do exercício físico em idosos pode se manifestar na melhora do humor, na redução das respostas fisiológicas ao estresse, em efeitos positivos na imagem corporal, no funcionamento cognitivo e na autoestima, além de melhora na qualidade do sono e maior satisfação com a vida.

A autopercepção de saúde também esteve associada com a ocorrência de sintomas depressivos entre os idosos de Pelotas, sendo os mais deprimidos aqueles que referiram ter pior saúde. Apesar de subjetiva, a autopercepção de saúde inclui as dimensões biológica, psíquica e social, e associa-se intimamente à ocorrência de depressão ${ }^{24}$. Este é um dos indicadores mais utilizados em estudos epidemiológicos e com idosos, sendo apontado como um bom indicador de bem-estar. A autoavaliação de saúde pode refletir um entendimento de mudanças biológicas e fisiológicas sutis que levam a perceber a própria saúde ou de forma mais positiva ou negativamente, podendo representar melhor o estado de saúde do que medidas objetivas ${ }^{40,41}$. Justifica-se sua forte associação com sintomas depressivos uma vez que a perda das funções cognitiva, sexual e laboral, a diminuição das relações sociais e o sentimento de invalidez, entre outros elementos, influenciam tanto para uma pior percepção de saúde como na ocorrência de sintomas depressivos ${ }^{42}$.

Os idosos com incapacidade funcional também apresentaram maior prevalência de sintomas depressivos, resultado também observado em outros estudos ${ }^{8,11,29,33}$. Contudo, um estudo de revisão sistemática e meta-análise conduzido por Cole e Dendukuri ${ }^{9}$ apontou para a existência de uma relação bidirecional de causa e efeito nesta associação. Isto porque o desenvolvimento de alguma limitação para realizar as atividades cotidianas pode gerar um sentimento negativo, o que pode levar a episódios depressivos. Da mesma forma, a redução do prazer nas atividades cotidianas, que é um dos sintomas da depressão, pode diminuir a capacidade de execução destas atividades, tornando-se extremamente difíceis.

A situação conjugal, o uso de álcool, o tabagismo e a presença de doenças crônicas não estiveram associadas, no modelo ajustado, com a 
ocorrência de sintomas depressivos em idosos neste estudo. De acordo com o modelo teórico de análise utilizado, fatores de confusão existentes entre estas características e os sintomas depressivos podem ser uma explicação plausível para as perdas de associação após ajuste. A associação entre situação conjugal e sintomas depressivos é inconsistente na literatura, de modo que muitos estudos apontam para resultados conflitantes ${ }^{9}$. Já a relação entre o desfecho e o consumo de álcool pode ser um pouco mais complexa. Alguns estudos apontam que o uso leve ou moderado de álcool pode ter repercussão positiva sobre a saúde mental ${ }^{25,34}$, enquanto que o consumo abusivo, altamente relacionado com piores desfechos em saúde de um modo geral, não apresenta associação com a ocorrência de sintomas depressivos entre idosos ${ }^{6}$. Além disso, a variável sobre uso de álcool incluída no modelo limita-se a informação de qualquer uso de álcool nos últimos 30 dias, sem possibilidade de especificação de quantidade e/ou frequência de uso. Em relação à associação entre morbidades e sintomas depressivos, esta associação pode ter perdido significância estatística após inclusão da incapacidade funcional no modelo multivariável, de modo que os idosos com mais morbidades incapacitantes podem apresentar maior prevalência de sintomas depressivos do que aqueles com doenças crônicas controladas. Com relação à associação entre tabagismo e sintomas depressivos, o valor inferior do IC $95 \%$ $(0,99-1,90)$ esteve no limite da significância estatística, indicando falta de poder estatístico para avaliar esta associação.

Este estudo utilizou para a definição do desfecho um instrumento de triagem, que oferece como resultado uma medida baseada na presença de sintomas depressivos, útil para rastrear casos na população. No entanto, o instrumento não substitui o diagnóstico baseado em entrevista clínica, realizada por psicólogos e psiquiatras. Além disso, a depressão é um episódio flutuante, portanto, os achados apontam para um momento - neste caso, refere-se à última semana. Isto pode ser um dos fatores que explicam a variações nas prevalências e em seus fatores associados nos diferentes estudos realizados em diversos países.

Entre as limitações, destaca-se o possível viés de causalidade reversa, inerente aos estudos transversais, que restringe algumas das associações encontradas no sentido de não ser possível estabelecer relações de causalidade entre as exposições e o desfecho pela ausência de temporalidade entre a ocorrência dos eventos de interesse, como pode observado no trabalho atual, atividade física, autopercepção de saúde e capacidade funcional. Além disso, estudos transversais em idosos também estão sujeitos ao viés de sobrevivência, o que pode levar a uma subestimação das associações. Outra possível limitação deve-se ao fato de que a validação do ponto de corte da GDS-10 foi realizada em uma amostra ambulatorial e não em uma amostra populacional. No entanto, deve-se destacar que as propriedades de sensibilidade e especificidade do instrumento foram comparadas com entrevista diagnóstica estruturada. Ressalta-se que não são esperadas diferenças entre os sintomas depressivos em indivíduos que foram avaliados em serviços de saúde ou que estão na comunidade. A diferença esperada será na prevalência destes sintomas, que tradicionalmente é maior em indivíduos nos serviços de saúde do que na comunidade. A prevalência da doença não altera a sensibilidade e a especificidade do teste.

Como vantagens, este estudo teve base populacional, realizado através de inquérito domiciliar, com baixo percentual de perdas e recusas se comparado a outros inquéritos populacionais. A literatura brasileira carece de estudos desta magnitude, de base populacional com amostra representativa e, principalmente, cuja população alvo seja os idosos. Sobretudo, a contribuição principal deste trabalho é fomentar a reflexão sobre a importância da avaliação dos sintomas depressivos em idosos e seus determinantes. Desta forma, espera-se evidenciar a necessidade de que profissionais e serviços de saúde realizem condutas e ações de rastreamento de forma sistemática, de forma a oferecer tratamento e cuidados para a população idosa. 


\section{Colaboradores}

N Hellwig e E Tomasi participaram de todas as etapas deste estudo, incluindo a idealização da proposta de pesquisa, a elaboração do projeto, a análise e interpretação dos dados e a redação do manuscrito. TN Munhoz colaborou na elaboração do projeto, interpretação dos resultados e redação do manuscrito.

\section{Agradecimentos}

Ao apoio financeiro da Coordenação de Aperfeiçoamento de Pessoal de Nível Superior (CAPES), com recursos provenientes do Programa de Excelência Acadêmica (PROEX).

\section{Referências}

1. Lebrão ML. O envelhecimento no Brasil: aspectos da transição demográfica e epidemiológica. Saúde Coletiva 2007; 4(17):135-140

2. Instituto Brasileiro de Geografia e Estatística (IBGE). Censo Demográfico. Rio de Janeiro: IBGE; 2010.

3. Garrido R, Menezes PR. O Brasil está envelhecendo: boas e más notícias por uma perspectiva epidemiológica. Rev Bras Psiquiatr 2002; 24(1):3-6.

4. Santos KT, Fernandes MH, Reis LA, Coqueiro RS, Rocha SV. Depressive symptoms and motor performance in the elderly: a population based study. Rev Bras Fisioter 2012; 16(4):295-300.

5. Reeves WC, Strine TW, Pratt LA, Thompson W, Ahluwalia I, Dringra SS, McKnight-Eily LR, Harrison L, D'Angelo DV, Williams L, Morrow B, Gould D, Safran MA; Centers for Disease Control and Prevention (CDC). Centers for Disease Control and Prevention (CDC). Mental Illness surveillance among adults in the United States. MMWR Surveill Summ 2011; 60(3):1-29.

6. Pinho MX, Custódio O, Makdisse M. Incidence of depression and associated factors among elderly community-dwelling people: a literature review. Rev Bras Geriatr Gerontol 2009; 12(1):123-140.

7. Reppermund S, Brodaty H, Crawford JD, Kochan NA, Slavin MJ, Trollor JN, Draper B, Sachdev PS. The relationship of current depressive symptoms and past depression with cognitive impairment and instrumental activities of daily living in an elderly population: the Sydney Memory and Ageing Study. J Psychiatr Res 2011; 45(12):1600-1607.

8. Castro-Costa E, Lima-Costa MF, Carvalhais S, Firmo JO, Uchoa E. Factors associated with depressive symptoms measured by the 12-item General Health Questionnaire in community-dwelling older adults (The Bambui Health Aging Study). Rev Bras Psiquiatr 2008; 30(2):104-109.

9. Cole GC, Dendukuri N. Risk factors for depression among elderly community subjects: a systematic review and meta-analysis. Am J Psychiatry 2003; 160(6):11471156.

10. Djernes JK. Prevalence and predictors of depression in populations of elderly: a review. Acta Psychiatr Scand 2006; 113(5):372-87.

11. Blay SL, Andreoli SB, Fillenbaum GG, Gastal FL. Depression Morbidity in Later Life: Prevalence and Correlates in a Developing Country. Am J Geriatr Psychiatry 2007; 15(9):790-9.

12. Yesavage JA, Brink TL, Rose TL, Lum O, Huang V, Adey M, Leirer VO. Development and validation of a geriatric depression screening scale: a preliminary report. $J$ Psychiatr Res 1982; 17(1):37-49.

13. Almeida OP, Almeida SA. Short versions of the geriatric depression scale: a study of their validity for the diagnosis of a major depressive episode according to ICD-10 and DSM-IV. Int J Geriatr Psychiatry 1999; 14(10):858-865

14. Matsudo S, Araújo T, Marsudo V, Andrade D, Andrade E, Braggion G. Questinário internacional de atividade física (IPAQ):estudo de validade e reprodutibilidade no Brasil. Rev bras ativ fís saúde 2001; 6(2):5-18.

15. Lino VTS, Pereira SRM, Camacho LAB, Ribeiro Filho ST, Buksman S. Adaptação transcultural da Escala de Independência em Atividades da Vida Diária (Escala de Katz). Cad Saude Publica 2008; 24(1):103-112. 
16. Barros AJD, Hirakata VN. Alternatives for logistic regression in cross-sectional studies: an empirical comparison of models that directly estimate the prevalence ratio. BMC Medical Research Methodology 2003; 3(1):113.

17. Victora CG, Huttly SR, Fuchs SC, Olinto MT. The role of conceptual frameworks in epidemiological analysis: a hierarchical approach. Int J Epidemiol 1997; 26(1):224-227

18. Barcelos-Ferreira R, Izbicki R, Steffens DC, Bottino CMC. Depressive morbidity and gender in community-dwelling Brazilian elderly: systematic review and meta-analysis. Int Psychogeriatr 2010; 22(5):712-726.

19. Alexandrino-Silva C, Alves TF, Tófoli LF, Wang YP, Andrade LH. Psychiatry - life events and social support in late life depression. Clinics 2010; 66(2):233-238.

20. Costa E, Barreto SM, Uchoa E, Firmo JO, Lima-Costa MF, Prince M. Prevalence of International Classification of Diseases, 10th Revision common mental disorders in the elderly in a Brazilian community: The Bambui Health Ageing Study. Am J Geriatr Psychiatry 2007; 15(1):17-27.

21. Nicolosi GT, Falcao DV, Batistoni SS, Lopes A, Cachioni M, Neri AL, Yassuda MS. Depressive symptoms in old age: relations among sociodemographic and self-reported health variables. Int Psychogeriatr 2011; 23(6):941-949.

22. Hoffmann EJ, Ribeiro F, Farnese JM, Lima EWB. Depressive symptoms and associated factors among elders dwelling in a community in the North of Minas Gerais state, Brazil. J Bras Psiquiatr 2010; 59(3):190-197.

23. Reichert CL, Diogo CL, Vieira JL, Dalacorte RR. Physical activity and depressive symptoms in community-dwelling elders from southern Brazil. Rev Bras Psiquiatr 2011; 33(2):165-70.

24. Maciel ACC, Guerra RO. Prevalence and associated factors of depressive simptomatology in elderly residents in the Northeast of Brazil. J Bras Psiquiatr 2006; 55(1):26-33.

25. Park JH, Kim KW, Kim MH, Kim MD, Kim BJ, Kim SK, Kim JL, Moon SW, Bae JN, Woo JI, Ryu SH, Yoon JC, Lee NJ, Lee DY, Lee DW, Lee SB, Lee JJ, Lee JY, Lee CU, Chang SM, Jhoo JH, Cho MJ. A nationwide survey on the prevalence and risk factors of late life depression in South Korea. J Affect Disord 2012; 138(1-2):34-40.

26. Wang JK, Su TP, Chou P. Sex differences in prevalence and risk indicators of geriatric depression: the Shih-Pai community-based survey. J Formos Med Assoc 2010; 109(5):345-353.

27. Navarro B, Andres F, Parraga I, Morena S, Latorre JM, Lopez-Torres J. Approach to major depression in old people. Int Psychogeriatr 2010; 22(5):733-738.

28. Yunming L, Changsheng C, Haibo T, Wenjun C, Shanhong F, Yan M, Yongyong X, Qianzhen H. Prevalence and risk factors for depression in older people in Xi'an China: a community-based study. Int J Geriatr Psychiatry 2012; 27(1):31-39.

29. Millan-Calenti JC, Maseda A, Rochette S, Vazquez GA, Sanchez A, Lorenzo T. Mental and psychological conditions, medical comorbidity and functional limitation: differential associations in older adults with cognitive impairment, depressive symptoms and co-existence of both. Int J Geriatr Psychiatry 2011; 26(10):1071-1079.
30. Garcia-Pena C, Wagner FA, Sanchez-Garcia S, Juarez-Cedillo T, Espinel-Bermudez C, Garcia-Gonzalez JJ, et al. Depressive symptoms among older adults in Mexico City. J Gen Intern Med 2008; 23(12):1973-1980.

31. Piccinelli M, Wilkinson G. Gender differences in depression. Critical review. Br J Psych 2000; 177:486-492.

32. Halbreich U. Role of estrogen in postmenopausal depression. Neurology 1997; 48(7):16-20.

33. Carvalhais SM, Lima-Costa MF, Peixoto SV, Firmo JO, Castro-Costa E, Uchoa E. The influence of socio-economic conditions on the prevalence of depressive symptoms and its covariates in an elderly population with slight income differences: the Bambui Health and Aging Study (BHAS). Int J Soc Psychiatry 2008; 54(5):447-456

34. Li N, Pang L, Chen G, Song X, Zhang J, Zheng X. Risk factors for depression in older adults in Beijing. Can J Psychiatry 2011; 56(8):466-473.

35. Lorant V, Deliège D, Eaton W, Robert A, Philippot P, Ansseau M. Socio-economic inequalities in depression: a meta-analysis. Am J Epidemiol 2003; 157(2):98-112.

36. Kessler RC, Aguilar-Gaxiola S, Alonso J, Chatterij S, Lee S, Ormel J, Ustün TB, Wang PS. The global burden of mental disorders: an update from the WHO World Mental Health (WMH) surveys. Epidemiol Psichiatr Soc 2009; 18(1):23-33

37. Gazalle FK, Lima MS, Tavares BF, Hallal PC. Sintomas depressivos e fatores associados em população idosa no Sul do Brasil. Rev Saude Publica 2004; 38(3):365-367.

38. Mather AS, Rodriguez C, Guthrie MF, McHarg AM, Reid IC, McMurdo ME. Effects of exercise on depressive symptoms in older adults with poorly responsive depressive disorder: randomized controlled trial. $\mathrm{Br} J$ Psychiatry 2002; 180:411-415.

39. Guimarães JMN, Caldas CP. A influência da atividade física nos quadros depressivos de pessoas idosas: uma revisão sistemática Rev bras epidemiol 2006; 9(4):481492.

40. Idler EL, Benyamini Y. Self-rated health and mortality: a review of twenty-seven community studies. $J$ Health Soc Behav 1997; 38(1):21-37.

41. Mossey JM, Shapiro E. Self rated health: a predictor of mortality among the elderly. Am J Public Health 1982; 72(8):700-708

42. Lima-Costa MF, Firmo JOA, Uchoa E. Differences in self-rated health among older adults according to socioeconomic circumstances: the Bambuí Health and Aging Study. Cad Saude Publica 2005; 21(3):830-839.

Artigo apresentado em 27/09/2015

Aprovado em 26/04/2016

Versão final apresentada em 28/04/2016 